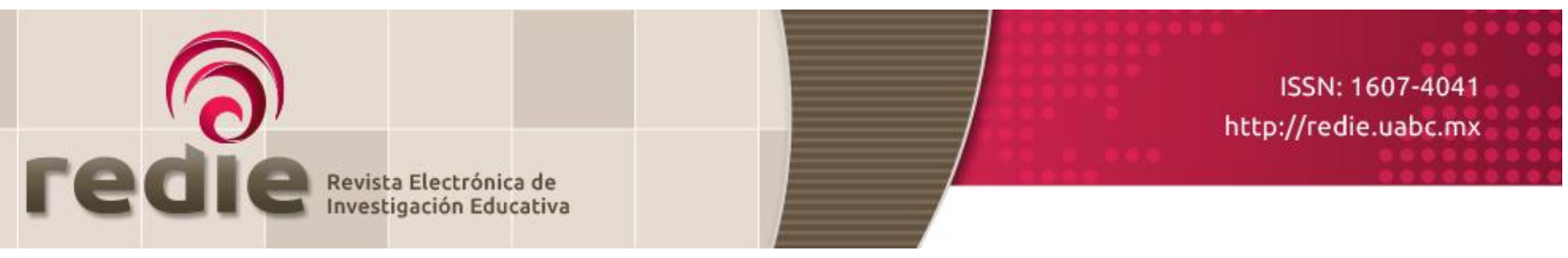

Vol. 19, Núm. 4, 2017

\title{
Evaluación de la competencia digital docente en la Comunidad Autónoma de Aragón
}

\section{Assessment of Digital Competence in Teachers in the Autonomous Community of Aragon}

\author{
José María Falcó Boudet (*) chema.falco@unizar.es \\ (*) Universidad de Zaragoza \\ (Recibido: 19 de abril de 2016; Aceptado para su publicación: 13 de junio de 2016)
}

Cómo citar: Falcó, J. M. (2017). Evaluación de la competencia digital docente en la comunidad autónoma de Aragón. Revista Electrónica de Investigación Educativa, 19(4), 73-83. https://doi.ora/10.24320/redie.2017.19.4.1359

\section{Resumen}

El presente artículo describe y analiza el nivel de la competencia digital docente en el profesorado de enseñanza media de la comunidad autónoma de Aragón (España). Se parte de los estándares fijados por organizaciones internacionales y por el Ministerio de Educación español para definir las dimensiones que la forman y para fijar los dos componentes que se estudian: conocimiento técnico y aplicación didáctica. Los datos se recogieron mediante un cuestionario diseñado con este propósito. Los resultados muestran que los profesores reconocen la aportación que las TIC pueden hacer al proceso de enseñanza-aprendizaje pero que, aunque tienen un nivel de desempeño medio para uso personal (búsqueda de información, utilización de herramientas más comunes), tienen un nivel bajo en el aprovechamiento didáctico. Los resultados evidencian la necesidad de fortalecer la formación inicial de los docentes y promover programas de formación permanente para el desarrollo de esta competencia.

Palabras clave: Tecnología educativa, Enseñanza media, Competencia Digital Docente.

\section{Abstract}

This paper describes and analyzes the level of digital competence in high school teachers in the autonomous community of Aragon (Spain). It takes the standards set by international organizations and the Spanish Ministry of Education as a basis to define the dimensions of digital competence and the two aspects studied: technical knowledge and educational application. Data was collected through a purposebuilt questionnaire. The results show that teachers recognize the contribution that ITC can make to the teaching-learning process, but that, despite an average level of performance for personal use (searching for information, using the most common tools), teachers' application of ITC to education is poor. The results highlight the need to strengthen teachers' initial training and promote continuing education programs to develop competence. 


\section{Introducción}

La incorporación de las Tecnologías de la Información y la Comunicación (TIC) al ámbito educativo guarda relación con dos aspectos: la presencia de estas herramientas en la realidad que estudiantes y docentes viven fuera del aula y la utilización de sus capacidades de buscar, crear, comunicar y compartir conocimientos en la mejora del proceso de enseñanza-aprendizaje. Los profesores tienen que integrar necesidades sociales -como aprender a hacer un buen uso de las TIC evitando una utilización adictiva o compulsiva- con necesidades profesionales, como el desafío de renovar las metodologías didácticas y con requerimientos legales, como la exigencia de la Ley Orgánica de Educación (LOE) y la Ley Orgánica para la Mejora de la Calidad Educativa (LOMCE) de educar por competencias para que los alumnos adquieran, entre otras, la competencia digital.

Sin embargo, pese a la presencia de ordenador o pizarra digital en todas las aulas, y a la reciente proliferación de los netbook en casi todos los centros escolares, en la realidad el profesorado utiliza las TIC para planificar su enseñanza, pero no para crear "ambientes enriquecidos de aprendizaje en los que estas tecnologías estén plenamente integradas" (Almerich, Suárez, Jornet y Orellana, 2011). Los usos principales son básicos: proporcionar orientaciones que faciliten el aprendizaje de los alumnos, como apoyo a la exposición oral de los contenidos o mostrar ejemplos de productos a realizar, y son pocos los docentes que las utilizan para plantear actividades donde Internet sea fuente de conocimiento y lugar de expresión o para desarrollar actividades en colaboración con estudiantes de otros centros (Vargas, Chumpitaz, Suárez y Badía, 2014). También entre los docentes universitarios "su introducción es marginal, más centrado en usos tradicionales que socioconstructivistas del aprendizaje, poco frecuentes de utilización, de usos para actividades tradicionales" (Cabero, 2014, p. 2).

Рara que se produzca una integración educativa efectiva de las TIC en las aulas es necesario que el profesorado las valore como un elemento esencial en el proceso de enseñanza-aprendizaje. No basta con conocer o incluso dominar los medios digitales. Es necesario poseer "nuevas competencias profesionales que garanticen tanto el saber como el saber hacer, el saber estar y el hacer saber en y con TIC" (Tejada, 2009, p. 12). Diversos autores han definido esta competencia profesional como la Competencia digital docente $(C D D)$.

\subsection{Competencia Digital Docente}

Krumsvik (2009) define la Competencia Digital Docente (CDD) como el uso de las TIC para enseñar y aprender con criterios didácticos y pedagógicos y con conciencia ética y moral. Castañeda (2015) considera que es "lo que debe saber un profesor para enseñar con tecnologías", y Lázaro y Gisbert (2015) la definen como la capacidad del profesorado de poseer un nivel de competencia digital que le permita utilizar la tecnología con eficacia, de forma adecuada y adaptada a sus estudiantes y a los aprendizajes que éstos deben conseguir.

Diversos organismos internacionales han elaborado propuestas de estándares para organizar los saberes y habilidades que los profesores con CDD deberían dominar: European pedagogical ICT Licence (EPICT), NETS-S (ISTE, 2008), Estándares de la competencia TIC para docentes (UNESCO, 2008), las Competencias TIC para docentes (Ministerio de Educación de Chile, 2011) o el "Marco común de competencia digital docente" (INTEF, 2103) son algunos ejemplos. El modelo propuesto por la UNESCO marca tres etapas: 1) alfabetización tecnológica (competencias básicas en TIC), 2) profundización del conocimiento (conocimientos más avanzados y aplicados a problemas de la vida real) y 3) construcción del conocimiento (capacidad de crear nuevos conocimientos); y va paralelo al modelo de Adopción-Adaptación-ApropiaciónInnovación propuesto por Krumsvik (2009) que se traduce en la adquisición de habilidades digitales básicas (uso de herramientas TIC, alfabetización informacional y tecnológica); adquisición de la competencia didáctica con TIC (utilizar la tecnología al servicio de la pedagogía) y la creación de estrategias de aprendizaje (capacidad de utilizar las tıc para seguir alcanzando nuevos objetivos).

Varios autores han hecho un análisis de las dimensiones o subcompetencias de la CDD. Tanto Silva, Gros y Garrido (2006) como Almerich, Suárez, Belloch y Orellana (2010) identifican dos formas de abordar los 
estándares, una centrada en las competencias tecnológicas y otra en las pedagógicas. Silva et al. (2006) las agrupan en seis dimensiones: 1) Manejo y uso operativo de hardware y software, 2) Diseño de Ambientes de Aprendizaje, 3) Vinculación tic con el currículo, 4) Evaluación de recursos y aprendizaje, 5) Mejoramiento profesional, y 6) Ética y valores; mientras que Vargas et al. (2014) especifican que las competencias tecnológicas comprenden la habilidad del profesor en el uso de: 1) la tecnología básica, 2) las aplicaciones informáticas básicas, 3) las aplicaciones multimedia, 4) las presentaciones multimedia, 5) el software educativo y 6) el uso de Internet, e indican que las competencias pedagógicas incluyen:

(...) las habilidades en el uso de la tecnología para: a) orientar la enseñanza y el aprendizaje, b) organizar el aula, c) diseñar ambientes de aprendizaje, d) evaluar el progreso del alumno y su diversidad, e) desarrollar la práctica profesional, f) llevar a cabo proyectos de innovación y g) resolver problemas sociales, éticos, legales y humanos. (p. 364).

Lázaro y Esteve (2015), por su parte, añaden los componentes de las estrategias de aprendizaje y de formación o capacitación digital a los componentes tecnológico y pedagógico.

Para este estudio se han establecido seis dimensiones. Las cinco primeras coinciden con las áreas que propone el Marco común para la competencia digital docente (Instituto Nacional de Tecnologías Educativas y Formación del Profesorado, 2013, p. 11):

1. Información: Identificar, localizar, recuperar, almacenar, organizar y analizar la información digital, evaluando su finalidad y relevancia.

2. Comunicación: Comunicar en entornos digitales, compartir recursos a través de herramientas en línea, conectar y colaborar con otros a través de herramientas digitales; interactuar y participar en comunidades y redes; conciencia intercultural.

3. Creación de contenido: Crear y editar contenidos nuevos (textos, imágenes, vídeos, etc.); integrar y reelaborar conocimientos y contenidos previos, realizar producciones artísticas, contenidos multimedia y programación informática, saber aplicar los derechos de propiedad intelectual y las licencias de uso.

4. Seguridad: Protección personal, protección de datos, protección de la identidad digital, uso de seguridad, uso seguro y sostenible.

5. Resolución de problemas: Identificar necesidades y recursos digitales, tomar decisiones a la hora de elegir la herramienta digital apropiada, acorde a la finalidad o necesidad, resolver problemas conceptuales a través de medios digitales, resolver problemas técnicos, uso creativo de la tecnología, actualizar la competencia propia y la de otros.

6. Utilización de materiales en soporte digital con fines didácticos: Evaluar si distintos materiales en soporte digital (aplicaciones interactivas, infografías, videos, WebQuest...) adecuados para ser medios en el logro de los objetivos de aprendizaje, hayan sido creados o no con este propósito, y utilizar dichos materiales con fines didácticos.

El objetivo principal de este estudio es describir y analizar la CDD de los docentes de enseñanza media de la comunidad autónoma de Aragón. Las preguntas que guiaron la investigación fueron: ¿Qué recursos tecnológicos conocen los profesores de esta comunidad autónoma y cuál es el uso educativo que les dan?, ¿el tipo de centro educativo al que pertenecen, el área o el nivel en el que imparten, el sexo, la edad o la experiencia son variables que influyen en ese uso?

Las interrogantes se concretaron en los objetivos: 1) Conocer la concepción que tienen los docentes sobre el uso educativo de las TIC en el aula y, en particular, en el área que imparten; 2) Identificar el nivel de desarrollo de las capacidades en las diferentes subdimensiones de la CDD y conocer si éste se relaciona con su nivel o etapa de integración en el proceso de enseñanza-aprendizaje; 3) Analizar si la edad, el sexo, 
los años de experiencia profesional, el tipo de centro educativo o el área que imparten son factores que influyen en el uso de las TIC.

\section{Método}

La investigación es de carácter exploratorio con un enfoque cuantitativo, de diseño no experimental, que tiene el objetivo de describir la situación actual de la CDD de los profesores de enseñanza media de la comunidad autónoma de Aragón en centros sostenidos con fondos públicos. La muestra estuvo compuesta por 361 docentes, $59.66 \%$ mujeres y $41.34 \%$ hombres. La mayoría ejerce la docencia en las etapas de secundaria obligatoria (75.9\%) y bachillerato $(52.35 \%) ; 39.34 \%$ imparte en Formación Profesional, $1.94 \%$ en Educación de Adultos y $1.66 \%$ en Escuelas Oficiales de Idiomas. El $76 \%$ de los participantes trabaja en un centro público y el $24 \%$ en un centro concertado. La experiencia docente de la mayoría es superior a 10 años (31\% de 11 a 20 cursos y 35\% de más de 20 cursos). Un 17\% tiene una experiencia de entre 5 y 10 cursos y otro $17 \%$ de cuatro cursos o menos. El $49.6 \%$ de los encuestados tiene más 45 años, un $49.3 \%$ entre 26 y 45 años y un $1 \%$ menos de 25 . La tabla I recoge la composición de la muestra por especialidades.

Tabla I. Distribución por especialidades

\begin{tabular}{l|r}
\hline Especialidades & $\%$ \\
\hline Ámbito científico & 18.0 \\
Ámbito de las ciencias sociales: geografía, historia... & 11.9 \\
Ámbito ético filosófico & 2.5 \\
Educación Física & 2.5 \\
Educación Plástica & 4.4 \\
Idiomas & 16.3 \\
Lengua castellana y literatura & 12.2 \\
Matemáticas & 15.8 \\
Música & 2.5 \\
Tecnología & 8.6 \\
FP & 26.0 \\
Orientación & 3.6 \\
\hline
\end{tabular}

La técnica empleada fue la encuesta y los datos se recogieron a través de la aplicación de un cuestionario online contestado de forma voluntaria y anónima. El cuestionario está fundamentado en el modelo teórico de CDD expuesto anteriormente y estructurado en cuatro apartados: datos personales y laborales, valoración de la aportación didáctica de las TIC, niveles de uso y conocimiento y utilización didáctica de las TIC. Para su diseño se adaptaron algunas cuestiones utilizadas anteriormente (Cabero, Llorente y Marín, 2010; Domínguez, 2011; Prendes, 2010; Roblizo y Cózar, 2015) y se introdujeron otras de elaboración propia que se adecuaban a las variables que se querían medir. La versión final del cuestionario fue aprobada por dos expertos y validada a través de pruebas piloto.

El apartado de valoración de la aportación didáctica de las TIC consta de 3 ítems, 2 con una escala Likert de 6 grados (de $0=n o$ aportan nada, hasta 5=aportan mucho) para valorar la aportación a nivel general y en la didáctica de la especialidad, y un tercero sobre las dificultades que hay para utilizar las TIC en el aula.

El apartado de niveles de uso y conocimiento consta de 32 ítems agrupados en 5 dimensiones: aspectos tecnológicos, actuación sobre aspectos tecnológicos, aspectos comunicativos, aspectos sobre la gestión de datos y tareas y aspectos sobre la gestión de la información. La escala Likert, también de 6 puntos, va en este caso desde " $0=$ No sé qué es" hasta " $5=$ Lo hago mucho" o "lo desempeño con mucha soltura. Soy capaz de explicar a otros cómo hacerlo".

El último apartado, utilización didáctica de las TIC, consta de 28 ítems agrupados en 4 dimensiones: sobre el uso de aplicaciones diseñadas para educación, sobre el uso de aplicaciones diseñadas para educación, sobre la creación de materiales propios y sobre las actividades de creación de productos que se les 
propone a los alumnos, valorados también con escala Likert de 6 puntos.

Se ha realizado un análisis estadístico descriptivo univariable y análisis correlacional entre factores demográficos y laborales (sexo, edad, experiencia docente, titularidad del centro educativo, especialidad o etapa) y las distintas variables utilizadas para medir la CDD, así como entre las variables que miden el conocimiento en TIC a nivel personal con su utilización en el aula.

\section{Resultados}

Los resultados están estructurados en tres apartados, en el primero se describe la valoración que hacen los profesores de la utilidad didáctica de las TIC y su utilización en el aula; en el segundo se muestran los resultados sobre el dominio de los aspectos tecnológicos (utilización de medios, resolución de problemas técnicos y protección de datos y de la identidad personal), gestión de la información y la comunicación (buscar, seleccionar y organizar la información y utilización de las TIC como medio de comunicación), gestión de tareas docentes y creación de materiales, tanto por parte del docente como del alumno (crear materiales en diferentes soportes, compartir y respetar los derechos de autor); y en el tercer y último apartado se expone la situación en relación a la utilización de materiales en soporte digital.

Las variables sexo, tipo de centro (público o concertado) y nivel educativo (Secundaria, Bachillerato y Formación profesional ${ }^{1}$ ) no han supuesto una alteración relevante con relación a los resultados generales en ninguna de las secciones, ni tampoco las variables edad, experiencia y nivel. Sólo la variable "área impartida" provoca alguna pequeña alteración que se comentará en su sección.

\subsection{Aportación didáctica de las TIC}

Un $84.7 \%$ de los docentes considera que las TIC pueden aportar bastante o mucho a mejorar el proceso de enseñanza-aprendizaje. Sólo dos docentes $(0.56 \%)$ de los encuestados consideran que no aportan casi nada. La valoración media, en una escala de 0 a 5, es de 4.22 (SD=0.76). Esta valoración disminuye algo al valorar la aportación en su área concreta: el $77.7 \%$ considera que puede aportar bastante o mucho, con una valoración media de 4.08 (SD=0.81). En la figura 1 se aprecia la media de la valoración por áreas. Si bien era de esperar que la Educación Física fuera una de las áreas en las que los docentes menos valoran la aportación de las TIC, sorprende que Matemáticas sea una de las cuatro áreas en las que esta aportación está peor valorada. Uno de los profesores de dicha área reconoce que no le interesan los temas relacionados con las TIC y que desconoce su empleo didáctico, pero la mayoría de ellos aporta como razón que los equipos informáticos están obsoletos o que los programas y contenidos son inadecuados para sus propósitos.

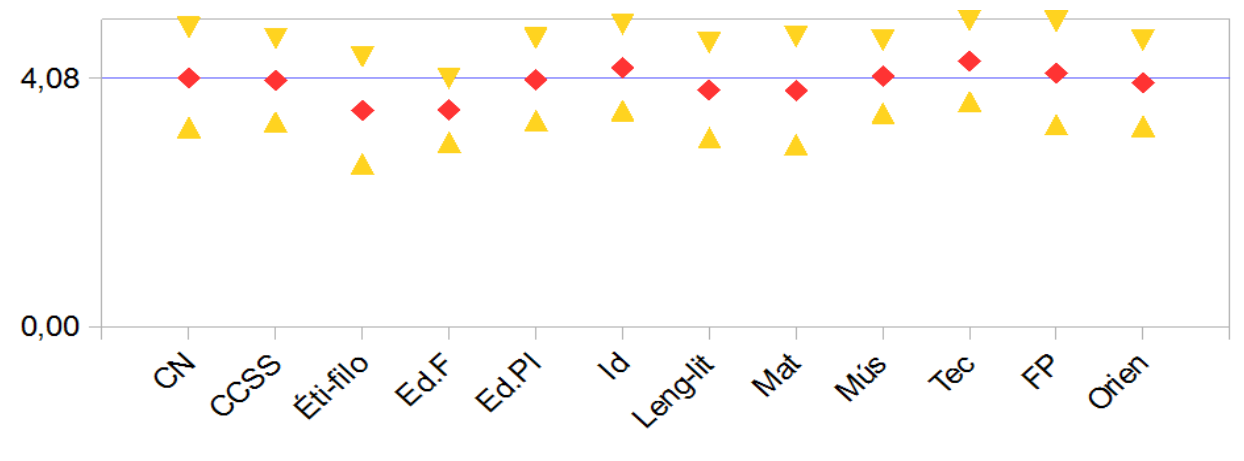

Figura 1. Valoración de las tıc al proceso de aprendizaje por áreas

\footnotetext{
${ }^{1}$ Las posibles variaciones en los niveles de Escuelas oficiales de idiomas y Educación de adultos no se consideran significativas dada la escasez de su presencia en la muestra.
} 
Además de su valoración se les preguntó por la utilización de las TIC en su práctica docente. Un 65\% manifestó utilizarlas habitualmente. Entre quienes no lo hacen la razón más repetida fue la falta de tiempo. Un $17.5 \%$ indicó que es debido a la extensión de los temarios. Otro $5 \%$ simplemente dijo que por "falta de tiempo". Un docente indicó que no tienen horas destinadas a creación de materiales; otro argumento destacado fue que los equipos son obsoletos (11\%) y faltan instalaciones adecuadas (13\%). También es relevante en relación con el centro el hecho de que la infraestructura no fuera la única razón: un 1\% (4 docentes) lo achacó a la falta de coordinación que facilite su utilización y 1.7\% (6 docentes) a la falta de iniciativa en su centro para potenciarlas.

También dieron argumentos de tipo didáctico, bien porque veían dificultad para integrarlas en el proceso de enseñanza-aprendizaje (5.5\%) o porque consideran que dificultan el esfuerzo y la iniciativa de los estudiantes (2.5\%). Un docente manifestó que "no aprenden mucho más. No sirven para enseñar los contenidos actuales" y una profesora del ámbito científico comentó que "el uso de las TIc empeora el desarrollo y el aprendizaje de la ortografía y la caligrafía".

No dominar los aspectos técnicos (1.66\%=6 docentes), su aplicación didáctica ( $1.1 \%=4$ docentes) o no dominar ninguna de las dos facetas ( $1 \%=3$ docentes) no fueron razones que mayoritariamente impidan la utilización didáctica de las Tıc.

\subsection{Nivel competencial en las cinco primeras dimensiones}

Aspectos tecnológicos. El nivel competencial de la dimensión tecnológica en su componente técnica es alto. Casi la totalidad de los docentes (99.45\%) es capaz de utilizar el ordenador, un $65.65 \%$ puede utilizar una pizarra digital sin ayuda y un $73.68 \%$ manifestó que es capaz de resolver sin ayuda problemas técnicos. Un $67 \%$ se preocupa por proteger el medio ambiente reciclando componentes y equipos. También son altos los niveles competenciales en cuanto a seguridad de los datos (80.33\% es capaz de hacerlo sin ayuda) y de protección de la identidad digital (75.35\%). El nivel competencial es algo más bajo en relación a la seguridad de los equipos: sólo un 50.4\% manifestó ser capaz de hacerlo sin ayuda, mientras un $14 \%$ manifestó no saber qué es y un 36\% indicó no saber cómo hacerlo o apenas saber. En cambio, el nivel del aspecto pedagógico de esta competencia es bajo, como se aprecia en la figura 2.

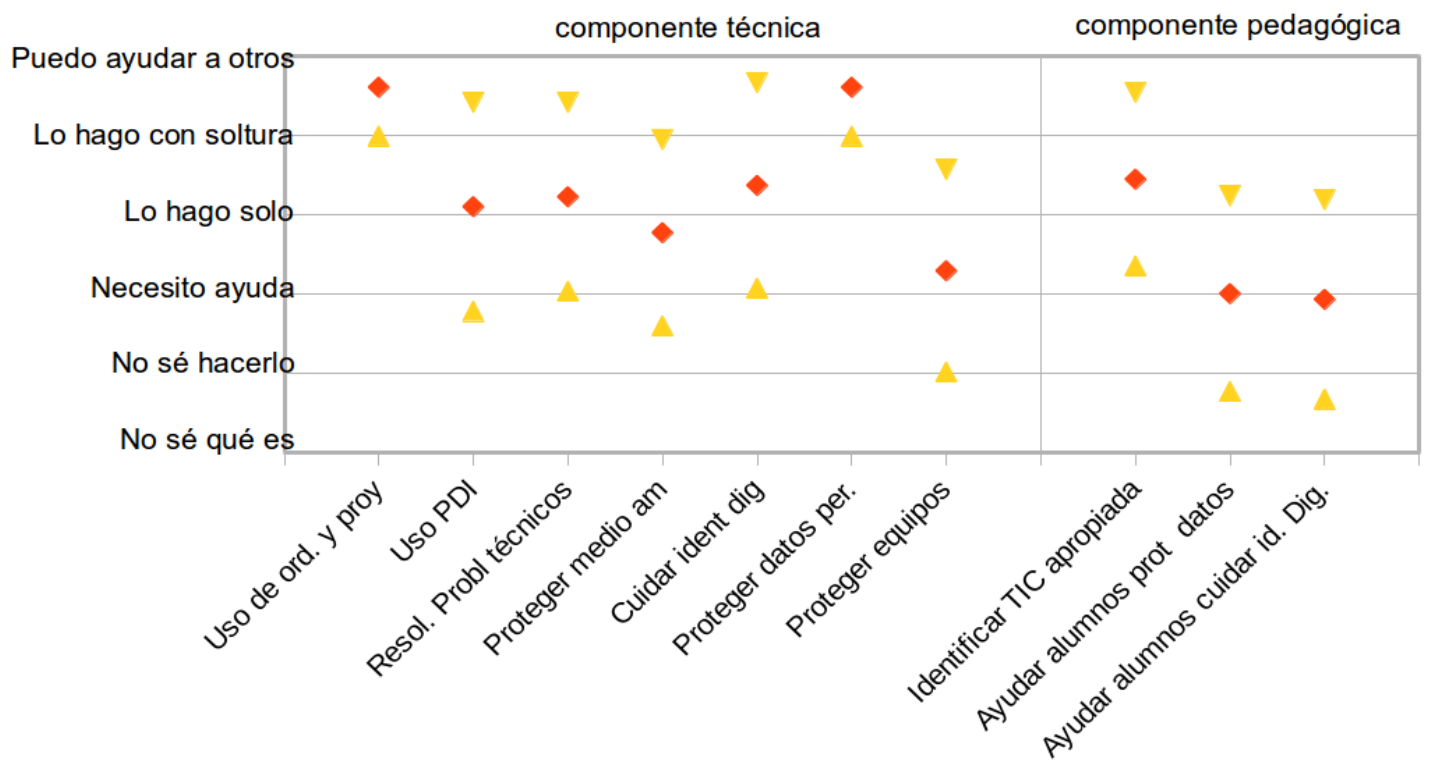

Figura 2. Dimensiones de la competencia tecnológica

Sólo un 35\% se preocupa de ayudar habitualmente a los alumnos a proteger sus datos personales, misma proporción que manifestó preocuparse por ayudarles a proteger su identidad digital. 15\% no sabe cómo 
ayudar a que protejan sus datos personales; $18 \%$ sabe, pero no lo hace nunca, y $32 \%$ lo hace muy esporádicamente. $18 \%$ no sabe cómo ayudar a que protejan su identidad digital; $17 \%$ sabe, pero no lo hace nunca, y $31 \%$ lo hace muy esporádicamente. El único aspecto con una valoración por encima de "lo hago sin ayuda" es el de ser capaz de elegir la herramienta TIC adecuada para potenciar o resolver una dificultad de aprendizaje. Se verá más adelante que este dato es contradictorio con el nivel de conocimiento de las herramientas TIC que manifestaron tener los profesores.

Existe una correlación significativa entre las componentes técnico y pedagógico de los niveles de la competencia en la protección de datos (Rho=0.68; $\mathrm{p}<0.05)$ y de la competencia en protección de la identidad personal (Rho=0.73; $p<0.05$ ), confirmando lo que estudios anteriores señalaban sobre la relación entre el conocimiento por parte del profesor de una determinada tecnología y su aplicación en el aula (Arancibia, Paz y Contreras, 2010; Puentes, Roig, Sanhueza y Friz, 2010).

Gestión de la información. Para describir el nivel competencial de la gestión de la información se consideran dos ejes: búsqueda y selección de la información relevante y clasificación y archivo de dicha información. Los docentes manifestaron tener un nivel alto en la componente técnica de la primera de ellas, ya que más de un $89 \%$ sabe navegar y utilizar las palabras clave para encontrar la información y seleccionar luego la que es fiable y relevante, con un 4.5 de valoración media (SD=0.72), pero el nivel de la componente pedagógica baja considerablemente: sólo un 54\% ayuda a sus alumnos a saber cómo encontrar y seleccionar información fiable y relevante, con un 3.5 de valoración media (SD=1.15). La correlación entre ambas componentes es moderada (Rho=0.49; $p<0.05$ ).

El nivel competencial para la clasificación y archivo de la información es claramente bajo en las dos componentes. Sólo 37\% utiliza habitualmente marcadores y $41 \%$ aplicaciones de gestión para clasificar y organizar la información, y apenas un $22.4 \%$ ayuda a que sus alumnos aprendan a realizar ambas tareas. En cambio, 33\% manifestó no saber qué es o cómo ayudar. La componente técnica dio una valoración media de 2.6 ( $\mathrm{SD}=1.82)$ y la pedagógica 2.2 ( $\mathrm{SD}=1.58)$, las dos por debajo de una utilización mínima. Entre ambas existe una correlación significativa (Rho=0.73; $p<0.05$ ).

Gestión de la comunicación. El nivel competencial de la componente técnica de la dimensión de comunicación es alto para usos básicos (3.9 en comunicación con alumnos y familias y 4.1 en comunicación con otros miembros del claustro). Pese a ello, 3\% de los docentes manifestó no saber qué es o cómo hacerlo. El nivel es medio para usos avanzados: 3.28 para comunicarse con docentes de otros centros o países (SD=1.25), 2.8 para compartir información a través de blogs o redes sociales $(S D=1.34)$ y se obtuvo un nivel bajo para usos avanzados: $65 \%$ no sabe qué es la "netetiqueta" o no sabe cómo utilizarla, y sólo 13\% manifestó utilizarla habitualmente (media=1.2; SD=1.56).

El nivel de la componente pedagógica de esta dimensión también es bajo. Los datos más significativos están recogidos en la tabla II, donde se aprecia un nivel de heterogeneidad alto, lo que indica niveles muy variados en los aspectos descritos en esta componente.

Tabla II. Nivel de la componente pedagógica de la dimensión de Comunicación

\begin{tabular}{l|c|c|c}
\hline & $\begin{array}{c}\text { Foro como herramienta } \\
\text { de apoyo al aprendizaje }\end{array}$ & $\begin{array}{c}\text { Actividades } \\
\text { online intercentros }\end{array}$ & $\begin{array}{c}\text { Enseñar y pedir } \\
\text { que usen 'netetiqueta' }\end{array}$ \\
\hline No sabe & $22.4 \%$ & $31.3 \%$ & $66.5 \%$ \\
No lo utiliza nunca & $32.7 \%$ & $38.5 \%$ & $16.3 \%$ \\
Uso esporádico & $19.4 \%$ & $16.6 \%$ & $7.8 \%$ \\
Uso habitual & $25.5 \%$ & $13.6 \%$ & $9.4 \%$ \\
\hline Med/ (SD) & $2.5 /(1.37)$ & $2.1 /(1.28)$ & \\
\hline
\end{tabular}

La correlación entre las componentes técnica y pedagógica de la utilización de la "netetiqueta" es muy alta (Rho=0.97; $\mathrm{p}<0.05$ ), lo que significa que aquellos docentes que tienen un nivel alto en este componente ético de la utilización de las TIC como medio de comunicación lo trasladan al proceso de enseñanza-aprendizaje. 
Gestión de tareas. Los niveles de los tres aspectos descritos en esta dimensión presentan una gran heterogeneidad. Los datos más significativos están recogidos en la tabla III. Se puede observar un nivel ligeramente superior en los docentes que ejercen en centros concertados frente a los que lo hacen en centros públicos.

Tabla III. Nivel de la dimensión Gestión de tareas

\begin{tabular}{|c|c|c|c|}
\hline & $\begin{array}{l}\text { Uso de ficheros para clasificar } \\
\text { datos de alumnos y familias }\end{array}$ & $\begin{array}{l}\text { Uso de aplicaciones } \\
\text { de gestión de tareas }\end{array}$ & $\begin{array}{l}\text { Uso de calendarios } \\
\text { y agendas digitales }\end{array}$ \\
\hline No sabe & $14.7 \%$ & $23.8 \%$ & $17.2 \%$ \\
\hline No lo utiliza nunca & $8.6 \%$ & $11.9 \%$ & $16.3 \%$ \\
\hline Uso esporádico & $13.9 \%$ & $23.0 \%$ & $21.9 \%$ \\
\hline Uso habitual & $62.9 \%$ & $41.3 \%$ & $44.6 \%$ \\
\hline Med/ (SD) & $3.5 /(1.46)$ & $2.9 /(1.58)$ & $3.1 /(1.50)$ \\
\hline Concertado & \begin{tabular}{l|r|} 
& 4.2 \\
\end{tabular} & 3.6 & \begin{tabular}{l|l} 
& 3.9 \\
\end{tabular} \\
\hline
\end{tabular}

Creación de materiales. El nivel competencial de la componente técnica de la dimensión de creación de materiales es alto para usos básicos, medio para otros usos más específicos y bajo para la utilización del copyleft. Esta situación se reproduce en la componente pedagógica, pero con unos niveles más bajos (ver figura 3). Se puede apreciar que los docentes de las áreas de Educación plástica, Música y Tecnología tienen un nivel superior a la media en algunos aspectos más técnicos.

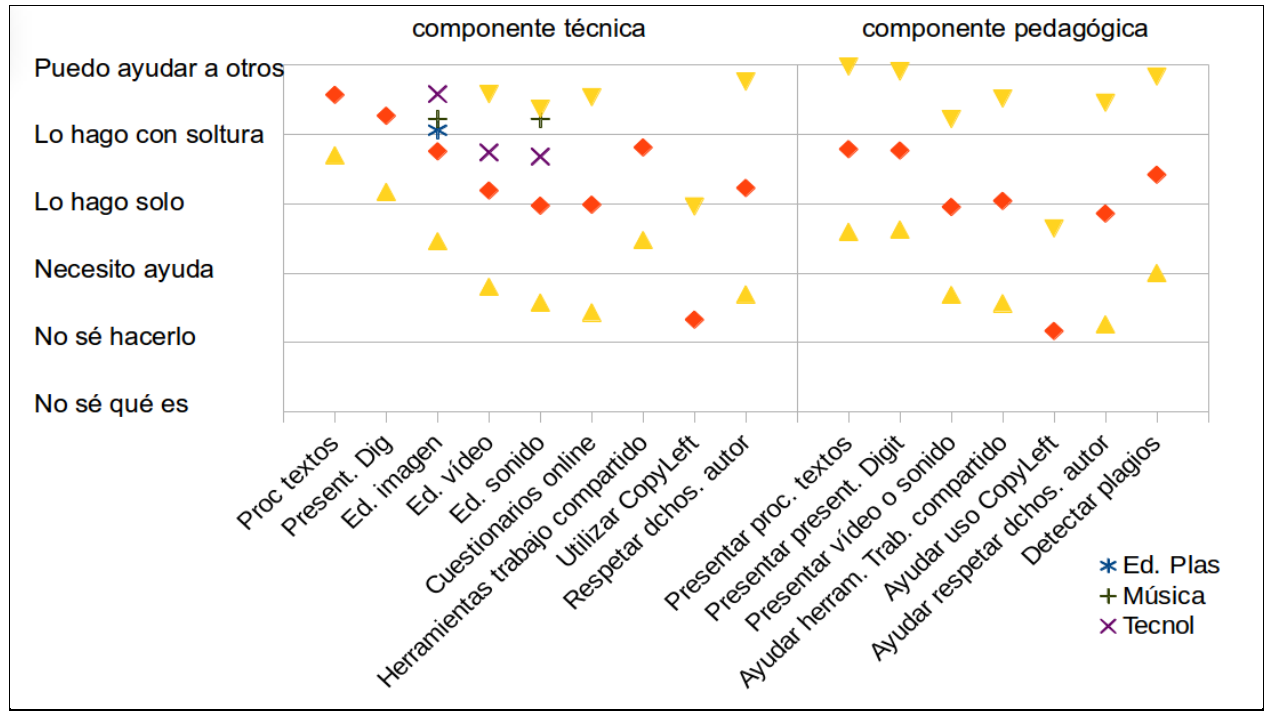

Figura 3. Aspectos de la dimensión de creación de materiales

Uso de materiales en formato digital. Pese a que en la segunda parte del cuestionario un $82.8 \%$ de los docentes manifestó ser capaz de identificar cuál es la herramienta TIC adecuada para potenciar o resolver una dificultad de aprendizaje, es significativo que de las diferentes herramientas que se propusieron sólo los videos y presentaciones digitales estuvieron por encima de la puntuación "Utilizo con bastante frecuencia" (4.19 y 4.10, respectivamente), mientras que otras que son específicas del ámbito educativo y que requieren una participación activa del alumnado, como las WebQuest apenas pasaron el nivel de conocimiento (media=1.88) o aquellas que son más adecuadas para provocar aprendizajes significativos, como los mapas mentales, sólo alcanzaron un nivel de utilización mínima (media=2.5). También obtuvieron puntuaciones un poco por encima de la utilización mínima aquellas tecnologías que necesitan una innovación metodológica para su uso, como los podcasts (2.34) o Google Maps (2.99); o aquellas que son emergentes, como las infografías (2.46). Por último, las herramientas diseñadas y creadas por el propio docente, para lo que se requiere un nivel de competencia técnica alto, obtuvieron una puntuación baja (1.76) (ver figura 4). 


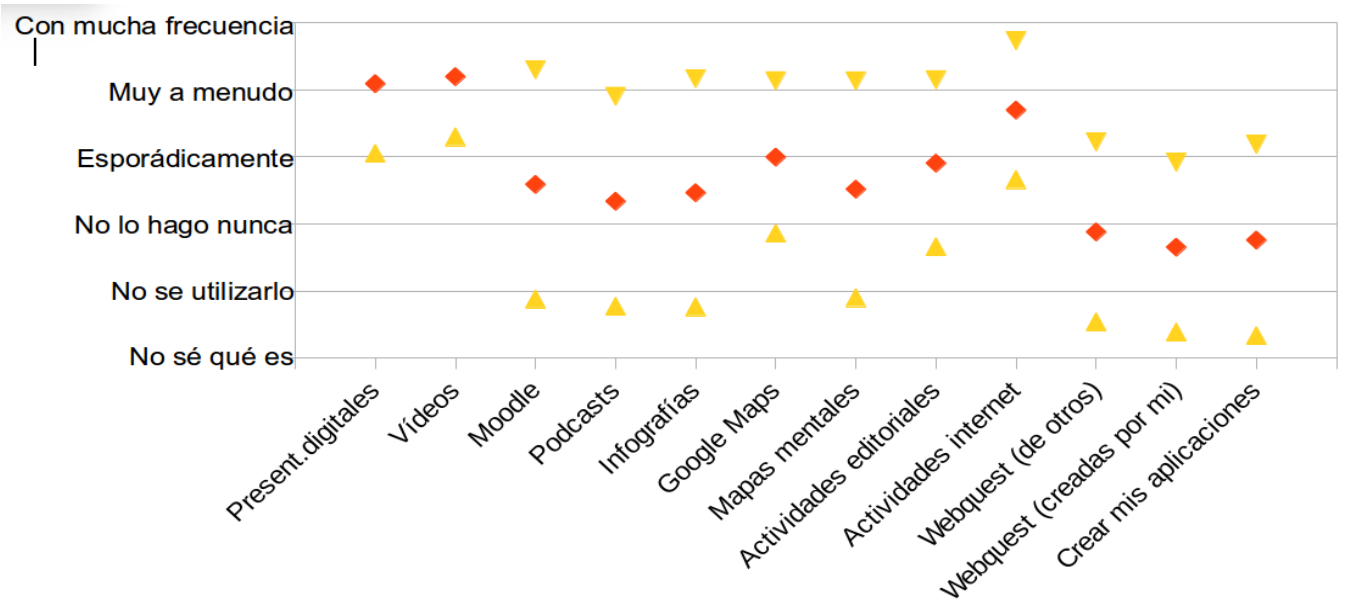

Figura 4. Niveles de uso de materiales en soporte digital

\section{Conclusiones}

Aunque se ha constatado una actitud positiva en el profesorado de enseñanza media de la comunidad autónoma de Aragón hacia el uso de las TIC en el proceso de enseñanza-aprendizaje -y que la mayoría de ellos utiliza las TIC-son pocos los que aprovechan su verdadero potencial haciendo que sean los alumnos quienes las utilicen de manera ética, responsable y cooperativa para que desarrollen su competencia digital. Salvo algunas excepciones ya señaladas, como la capacitación de los docentes de las áreas de Educación Plástica, Música o Tecnología para el uso de determinadas herramientas, las variables sexo, edad, experiencia, tipo de centro, etapa o área no influyen en los resultados. Esto lleva a pensar en que el desarrollo de la CDD tiene que ver con un componente personal y de compromiso profesional.

Los datos muestran el perfil de un docente que sabe buscar en Internet y seleccionar la información que necesita, que utiliza el correo para comunicarse, que utiliza videos para preparar sus clases, presentaciones digitales y algún otro material disponible en Internet o proporcionado por las editoriales y que potencia que sus alumnos busquen y seleccionen la información y elaboren presentaciones digitales. Este perfil competencial adolece de aspectos éticos (respeto a los derechos de autor, netetiqueta), sociales (utilización de foros y redes sociales, copyleft), es bajo en conocimientos técnicos (resolución de problemas técnicos, protección de los equipos, cuidado del medioambiente), en conocimientos de herramientas TIC y en la componente pedagógica, lo que confirma estudios previos sobre el escaso desarrollo de la CDD en el profesorado (Almerich et al., 2011; Sigalés, Mominó, Meneses y Badia, 2009).

Sin perder de vista las limitaciones de este estudio, con una muestra circunscrita a un territorio y a niveles de enseñanza específicos, se han encontrado evidencias de correlación entre conocimiento y aplicación didáctica, más fuerte en aquellos aspectos con una puntuación más baja, ya que los profesores que conocen determinados aspectos los trasladan a sus aulas. Los resultados de esta investigación demandan que los planes de estudios de los másteres en profesorado y los planes de formación permanente incorporen contenidos que logren el desarrollo de la competencia digital docente.

\section{Referencias}

Almerich, G., Suárez, J., Belloch, C. y Orellana, N. (2010). Perfiles del profesorado a partir del conocimiento de los recursos tecnológicos y su relación con el uso que hacen de estas tecnologías. Revista Complutense de Educación, 21(2), 247-269. Recuperado de http://revistas.ucm.es/index.php/RCED/article/view/15925 
Almerich. G., Suárez, J., Jornet, J. y Orellana, M. (2011). Las competencias y el uso de las Tecnologías de la Información y Comunicación por el profesorado: estructura dimensional. Revista Electrónica de Investigación Educativa, 13(1), 28-42. Recuperado de https://redie.uabc.mx/redie/article/view/269

Arancibia, M., Soto, C. P. y Contreras, P. (2010). Concepciones del profesor sobre el uso educativo de las tecnologías de la información y la comunicación (TIC) asociadas a procesos de enseñanza-aprendizaje en el aula escolar. Estudios Pedagógicos, 36(1), 23-51.

Cabero, J., Llorente, M. y Marín, D. (2010). Hacia el diseño de un instrumento diagnóstico de «competencias tecnológicas del profesorado» universitario. Revista Iberoamericana de Educación, 52(7), 112

Cabero, J. (2014). Formación del profesorado universitario en TIC. Aplicación del método Delphi para la selección de los contenidos formativos. Educación XXI,17 (1), 111-132. doi: 10.5944/educxX1.17.1.10707

Castañeda, L. (2015). Conferencia inaugural Congreso escola TIC 2015. Santiago de Compostela.

Domínguez, R. (2011). Formación, competencias y actitudes sobre las tic del profesorado de secundaria: un Instrumento de evaluación. RevistaEtic@net, 10. Recuperado de

http://www.ugr.es/ sevimeco/revistaeticanet/numero10/Articulos/Formato/articulo6.pdf

Gisbert, M. y Lázaro, J. L. (2015). La formación permanente en competencia digital docente y la mejora de la calidad del centro educativo desde la perspectiva de los docentes: un estudio de caso. New Approches in Educational Research, 4(2), 124-131. Recuperado de http://naerjournal.ua.es/article/viewFile/v4n2-7 $\not 157$

Instituto Nacional de Tecnologías Educativas y Formación del Profesorado. (2013). Marco común de competencia digital docente. Recuperado de http://educalab.es/documents/10180/12809/MarcoComunCompeDigiDoceV2.pdf/

Krumsvik, R. J. (2009). Situated learning in the network society and the digitised school. European Journal of Teacher Education, 32(2), 167-185.

Lázaro, J. L. y Gisbert, M. (2015). El desarrollo de la competencia digital docente a partir de una experiencia piloto de formación en alternancia en el Grado de Educación. EDUCAR, 51(2), 321-348. Recuperado de http://educar.uab.cat/article/view/v51-n2-lazaro-gisbert

Ministerio de Educación de Chile. (2011). Competencias y Estándares tıC para la profesión docente. Santiago de Chile: Autor.

Prendes, M.P. (2010). Competencias TIC para la docencia en la Universidad Pública española. Indicadores y propuestas para la definición de buenas prácticas. Programa de estudio y Análisis. Recuperado de http://www.um.es/competenciastic/instrumentos.html

Puentes, A., Roig, R., Sanhueza, S. y Friz, M. (2013, enero). Concepciones sobre las tecnologías de la información y la comunicación (TIC) y sus implicaciones educativas: Un estudio exploratorio con profesorado de la provincia de Nuble, Chile. Revista cTS, 8(22), 75-88. Recuperado de http://www.revistacts.net/files/Volumen\%208\%20-\%20N\%C3\%BAmero\%2022/SanhuezaDEF.pdf

Roblizo, M. J. y Cózar, R. (2015). Usos y competencias en TIC en los futuros maestros de Educación Infantil y Primaria: Hacia una alfabetización real para docentes. Píxel-Bit. Revista de Medios y Educación, 47. Recuperado de http://acdc.sav.us.es/ojs/index.php/pixelbit/article/view/225

Servicio de información comunitario sobre investigación y desarrollo. (2004). European Pedagogical ICT Licence. Recuperado de http://www.epict.org/info/about/ 
Sigalés, C., Mominó, J., Meneses, J. y Badia, A. (2009). La integración de Internet en la educación escolar española: Situación actual y perspectivas de futuro. Barcelona: Ariel.

Silva, J., Gros, B., Garrido, J. M. y Rodríguez, J. (2006). Estándares en tecnologías de la información y la comunicación para la formación inicial docente: situación actual y el caso chileno. Revista Iberoamericana de Educación, 38(3). Recuperado de http://rieoei.org/deloslectores/1391Silva.pdf

Tejada, J. (2009). Competencias docentes. Revista de Currículum y Formación de Profesorado, 13(2). Recuperado de http://digibug.ugr.es/bitstream/10481/7373/1/rev132COL2.pdf

UNESCO (2011). Estándares de competencia en TIC para docentes. Recuperado de http://www.eduteka.org/pdfdir/UNESCOEstandaresDocentes.pdf

Vargas, J., Chumpitaz, L., Suárez, G. y Badia, A. (2014). Relación entre las competencias digitales de docentes de educación básica y el uso educativo de las tecnologías en el aula. Revista de Currículum y Formación del Profesorado, 18(3), 361-377. Recuperado de http://www.ugr.es/ recfpro/rev183COL9.pdf 